\title{
Simulation of diffusion in a crowded environment
}

Cite this: Soft Matter, 2014, 10, 3597

Received 12th November 2013 Accepted 18th February 2014

DOI: $10.1039 / \mathrm{c} 3 \mathrm{sm} 52861 \mathrm{~h}$

www.rsc.org/softmatter

\author{
Piotr Polanowski and Andrzej Sikorski ${ }^{\star b}$
}

We performed extensive and systematic simulation studies of two-dimensional fluid motion in a complex crowded environment. In contrast to other studies we focused on cooperative phenomena that occurred if the motion of particles takes place in a dense crowded system, which can be considered as a crude model of a cellular membrane. Our main goal was to answer the following question: how do the fluid molecules move in an environment with a complex structure, taking into account the fact that motions of fluid molecules are highly correlated. The dynamic lattice liquid (DLL) model, which can work at the highest fluid density, was employed. Within the frame of the DLL model we considered cooperative motion of fluid particles in an environment that contained static obstacles. The dynamic properties of the system as a function of the concentration of obstacles were studied. The subdiffusive motion of particles was found in the crowded system. The influence of hydrodynamics on the motion was investigated via analysis of the displacement in closed cooperative loops. The simulation and the analysis emphasize the influence of the movement correlation between moving particles and obstacles.

\section{Introduction}

Fluid transport in a crowded environment plays a crucial role in a variety of fields: diffusion of lipids and proteins in cellular membranes, ${ }^{\mathbf{1}}$ water infiltration in porous media, ${ }^{2}$ transport in glasses ${ }^{3}$ and in supercooled liquids ${ }^{4}$ and diffusion of islands in metals. ${ }^{5}$ The dynamics in such systems is still far from being understood as the experiments indicate the diversity of dynamic behavior and even its origin are being discussed. ${ }^{6-14}$ The environment in living cells is a good example of such a system. It is mostly heterogeneous, because the motion of molecules is obstructed here by different kinds of lipids and proteins. Thus, it can be treated as a representative, interesting and challenging problem. ${ }^{15,16}$ Experimental studies concerning the motion of proteins and lipids in cells were performed using fluorescence correlation spectroscopy, ${ }^{17-20}$ pulsed field gradient $\mathrm{NMR}^{21}$ and single particle tracking (SPT) ${ }^{6,7,22-27}$ In many cases an anomalous diffusion was detected, i.e. the mean square displacement of objects $\left\langle\Delta r^{2}\right\rangle$ scaled with time $t$ as $\left\langle\Delta r^{2}\right\rangle \sim t^{\alpha}$, with $\alpha<1$. $^{6,28-30}$ On the other hand, a regular Brownian motion was also observed in such an environment, i.e. the scaling exponent $\alpha$ was equal to $1 .^{15}$ Beside changes of the dynamic behavior, a crowded environment can also change the rate of the association and adsorption of macromolecules. ${ }^{31}$ It should be pointed out that, recently, it was proven that the slowing down of motion in living cells had previously been overestimated. ${ }^{\mathbf{1 6}}$

Theoretical treatment of the motion of objects in a crowded environment also results in a subdiffusive behavior. ${ }^{32-37}$ One

\footnotetext{
${ }^{a}$ Department of Molecular Physics, Technical University of Łódż, 90-924 Łódż, Poland ${ }^{b}$ Department of Chemistry, University of Warsaw, Pasteura 1, 02-093 Warsaw, Poland. E-mail: sikorski@chem.uw.edu.pl; Fax: +48 22822 5996; Tel: +48 228220211
}

should remember that similar motion could be achieved by the influence of a geometrical confinement or forces. Computer simulations showed that this subdiffusive motion in the matrix of immobile obstacles has been characterized by the exponent $\alpha$ located in a narrow range between 0.697 (at the percolation threshold) and 1 (ref. 18, 38-42) while SPT experiments give values between 0.2 and $0.9 .{ }^{37}$ This kind of subdiffusive behavior can be obtained using several models and herein we focus our attention on two cases only. In the first one called continuous time random walks approach (CTRW), anomalous diffusion results from appropriate distribution of traps..$^{38-43}$ The second one corresponds to a well-known percolation problem, where anomalous diffusion is caused by the presence of the matrix of fixed impenetrable obstacles, which results in numerous dead ends. ${ }^{\mathbf{8} 28}$ The crowded environment in such cases can be modeled as a cluster of obstacles and this problem can be discussed using the theory of percolation. If the concentration of obstacles is lower than the percolation threshold, the diffusion should be anomalous (non-Fickian) only for a short time. If the concentration of obstacles reaches the percolation threshold, the diffusion becomes anomalous over the entire time range. ${ }^{28,30}$ The simulation studies of such systems were performed employing the molecular dynamics technique using coarse-grained and atomistic models. ${ }^{44-54}$ Moreover, one should remember that in reality the motion of a given molecule is correlated with the movement of neighboring molecules. The influence of traps and obstacles should also be taken into consideration. Thus, the questions how do the molecules move in a complex environment and how does the correlation of all elements in this motion influence their mobility are especially interesting in cell biology, soft matter physics and in many other areas. 
In this paper we have investigated the percolation problem with static obstacles within the frame of the Dynamic Lattice Liquid (DLL) model. ${ }^{55,56}$ The dynamics of liquid in a twodimensional crowded environment was an object of our investigation. The correlations in motion between elements of the entire system (moving elements, elements in traps or static obstacle elements) were taken into consideration. There is an important difference between the proposed herein treatment and other lattice models generally based on the concept of "an ant in the labyrinth". ${ }^{28,57}$ In this kind of model a single point ("an ant") travels on a lattice and methods that take into account jumps of mixture elements into vacancies (the vacancies are regarded as fluctuating free volume assuming that their size is comparable with that of the mobile elements) are employed. In these considerations the correlation and hydrodynamic effects are practically neglected. The present study allows us to include the cooperative motion of elements and to study the hydrodynamic properties of the system, which are apparently crucial for the diffusion in a crowded environment. . $^{58-60}$

\section{The DLL model and simulation conditions}

The DLL model is based on the concept of strictly cooperative motion of molecules in a dense system. ${ }^{55,56}$ The positions of beads representing molecules or larger objects are regarded as coinciding with lattice sites. For simplicity, the positions of the beads are limited to vertices of a quasi-crystalline lattice. All lattice sites may be occupied by beads so fully filled systems can be studied. It has been assumed that the system has some excess volume so each molecule has enough space to vibrate around its position defined by the lattice site. The beads, however, cannot easily move over a longer distance, because all neighboring lattice sites are occupied. Nevertheless, a longrange motion can take place in such systems in reality. The studies on the DLL model allow determination of the conditions required for molecular translations over distances exceeding the vibrational range. Each displacement of a molecule from the mean position is considered as an attempt of a movement to a neighboring lattice site. For simplicity, the directions of the attempts are assumed along the coordination lines of the lattice only, but they are independent and randomly distributed among the $q$ directions, where $q$ is the lattice coordination number. The DLL model fulfills the continuity equation and provides the correlated movements of 'molecules' as in a real liquid; it does not reproduce all properties known from the liquid mechanics but it is sufficient for studies of coarsegrained models. It is based on the model of liquid where molecules vibrate near quasi-localized points (staying in given lattice sites) sometime being involved in a motion correlated with neighbors resulting in a translation. The simulations were performed at the highest possible density, which cannot be obtained using other models. Molecular dynamics simulations of models with a matrix of immobilized obstacles were usually performed for liquid concentration near 0 (ref. 46 and 51) but the total concentration (fluid + obstacles) in these studies did not exceed $0.6 .^{49}$ Properties generated by the DLL model reflected suitable dynamic behavior in several problems like simple liquid dynamics, ${ }^{55}$ polymer-polymer interdiffusion, ${ }^{56}$ reaction diffusion front problems, ${ }^{61}$ polymer solution dynamics $^{62}$ and dynamics of the ATRP. ${ }^{63-68}$

As depicted in Fig. 1, the DLL cooperative rearrangements on the lattice have a form of closed loops of displacements involving at least three molecules. In contrast to many other lattice models, DLL allows one to study lattice systems at the density $\rho=1$ (all lattice sites are occupied). Moreover, the dynamic properties, which it produces, are in good agreement with those established for liquids. ${ }^{55,56}$ We employed a triangular lattice, with periodic boundary conditions imposed along the $x$ and $y$ axes. Time was assumed to be a discrete variable in which the positions of all molecules were attempted to be updated simultaneously. At any moment each lattice node was occupied by exactly one molecule and to each molecule $k$ a unit vector $\boldsymbol{v}_{\boldsymbol{k}}$ was assigned that pointed randomly at one of the nearest neighboring sites. This vector represented the direction along which the molecule attempted to move to an adjacent site. Then, all closed loops determined by these vectors were identified. The molecules that did not belong to a closed loop consisting of at least three elements were immobilized at the given time step. The static obstacles in the system can be introduced in a very simple way within the frame of the DLL model - an element chosen randomly as an obstacle cannot take place in a cooperative loop in any case.

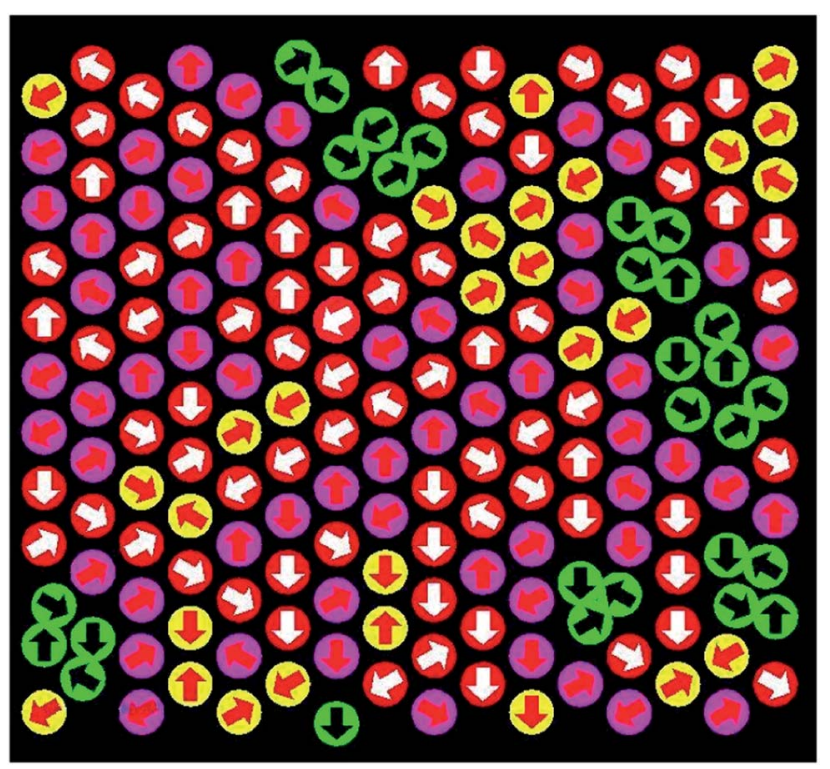

Fig. 1 The illustration of the vector field representing attempts of molecular displacements towards neighboring lattice sites as assumed in the DLL model. The marked areas represent various local situations: (1) elements (yellow) try to move in the opposite direction (an unsuccessful attempt), (2) an attempt of motion starts from an element (violet) that when moved would not be replaced by any of its neighbors (an unsuccessful attempt), and (3) each green element replaces one of its neighbors (successful attempts). 
Thus, the simulation process of a simple liquid using the DLL algorithm consisted of three steps:

(1) The generation of the random vector field of motion attempts with a single vector assigned to each bead in the athermal case. A unit vector pointed towards one of the nearestneighboring lattice sites. This is illustrated in Fig. 1.

(2) The selection of groups of vectors coinciding with contours of closed continuous paths (loops), indicating ways of possible successful cooperative rearrangements.

(3) The rearrangement of elements (beads) along these closed paths by displacing them to the neighboring sites (each bead replaces its neighbor).

The studies were performed in the simulation box $L \times L$ where $L=256$. It was shown previously within the frame of the regarded model that for the system larger than $L=64$ the statistic of cooperative loops of displacement did not depend on the size of the Monte Carlo box. ${ }^{55}$ In all cases static obstacles were generated randomly with the assumed probability. In all considered systems all lattice sites were occupied by immobile obstacles or mobile particles, which implied a dense system. Parallelism of the used algorithm allows monitoring of simultaneously more than 40000 trajectories for each density of obstacles. The trajectories extend over huge time windows consisting of typically $2.5 \times 10^{8}$ simulation steps near the percolation threshold. The concentration of the obstacles (each of them had a size of a lattice bead) in the system $c$ was defined as the ratio of the sites occupied by the obstacles to the total number of lattice sites in the simulation box $\left(c=N / L^{2}\right.$, where $N$ is the number of immobile obstacles in the system). Thus, the concentration of mobile elements (beads), i.e. molecules of liquid phase was $p=1-c$. The simulations were repeated many times and each simulation run was performed for a different matrix of randomly set obstacles (about 30 different matrices of obstacles) and the results were averaged over all runs for a given set of parameters.

\section{Results and discussion}

\section{A. General characteristics of the DLL model in the presence of immobile obstacles}

As we have already stated the considered system consists of objects located at vertices of a triangular lattice, while some randomly positioned vertices cannot move. Although, this kind of system seems to be very simple, immobile obstacles form clusters (i.e. groups in which immobile elements contact each other). The question arises about the size of clusters formed by these obstacles and their influence on the motion of mobile elements (liquid). Moreover, the explicit treatment of solvent molecules and their cooperative motion results in the momentum transport of the fluid and, therefore, it introduces hydrodynamic interactions. Hydrodynamic effects are modified by the presence of the immobilized obstacles. A simple analysis of hydrodynamic interactions in the regarded system was based on the monitoring of the motion in a cooperative loop, where the main quantity is the total probability of an elementary bead movement according to the mechanism proposed in the DLL model:

$$
P_{\mathrm{tot}}=\sum_{k=3}^{M} k P(k)
$$

where $k$ is the length while $M$ is the maximum length of the cooperative loop. The quantity $P(k)$ is adopted from ref. 62 and it plays a crucial role in the determination of dynamics of the investigated system. It can be regarded as a probability of " $k$ circuits" formation on a given lattice. This quantity can be analytically defined as: ${ }^{62}$

$$
P(k)=B k^{-h} \gamma^{k}
$$

where $B$ is a lattice dependent constant, $\gamma$ plays a role of an effective coordination number (called "connective constant") of the lattice, and $h$ is a positive exponent. The exponent $h$ depends on the dimensionality of space and is largely independent of the structure of the lattice. The theories predict the lower boundary for this exponent: $h \geq d / 2$, where $d$ is the dimensionality of space $^{67,68}$ (in the case of a triangular lattice these parameters were found as follows: $B=1.07 \pm 0.11, \gamma=0.710 \pm 0.003, h=$ $2.93 \pm 0.07$ (ref. 55)). This relationship has been derived for lattices, which (i) are homogeneous in the sense that the number of self-avoiding walks of $k$ steps is independent of the starting site, (ii) for each positive integer $k$, at least one $k$-step self-avoiding walk is possible, and (iii) the number of bonds leading out of any site is finite. In our case these assumptions are fulfilled for the case with no obstacles $(c=0)$ only, when the lattice is really homogeneous. ${ }^{55}$ We assumed that this quantity is independent of the system size, if the system was large enough; a similar assumption was made for the homogeneous case. ${ }^{55}$ Thus, the product $k \cdot P(k)$ is the probability that a bead takes place in a cooperative loop of the length $k$. In other cases, i.e. in the presence of obstacles our lattice system is not homogeneous because the points that are immobilized cannot be a starting point for cooperative loop. Fig. 2a presents the probability that a bead belongs to a cooperative loop of the length $k$ versus the length of the loop $k$. One can observe that the shape of the curves for the systems with obstacles $(c>0)$ is similar to that of the curve for $c=0$. This indicates the possibility of a universal scaling behavior. One can introduce the effective coordination number $\gamma(1-c)$ where $\gamma$ is the effective coordination number for the homogeneous case $(c=0)$ and $(1-c)$ is the liquid concentration. Fig. $2 b$ presents plots of the reduced quantities $\left(k \cdot P(k) /(1-c)^{k} v s\right.$. $\left.k\right)$, which results in the "collapse" of all curves into one. This behavior confirms the assumptions of the common behavior of homogeneous and inhomogeneous systems.

An analysis of the cooperative loop statistics directly relates to the distribution of waiting times. This analysis allows us to observe changes of hydrodynamic properties of the system with the increasing concentration of obstacles. It is shown in Fig. 2a that the cooperative loop is longer, the probability that a bead belongs to this loop decreases rapidly. One can also observe that an increase of the concentration of obstacles leads to a significant reduction of the probability of performing a long cooperative loop - the rate of this reduction increases with the loop length. The length of the cooperative loop decreases almost 

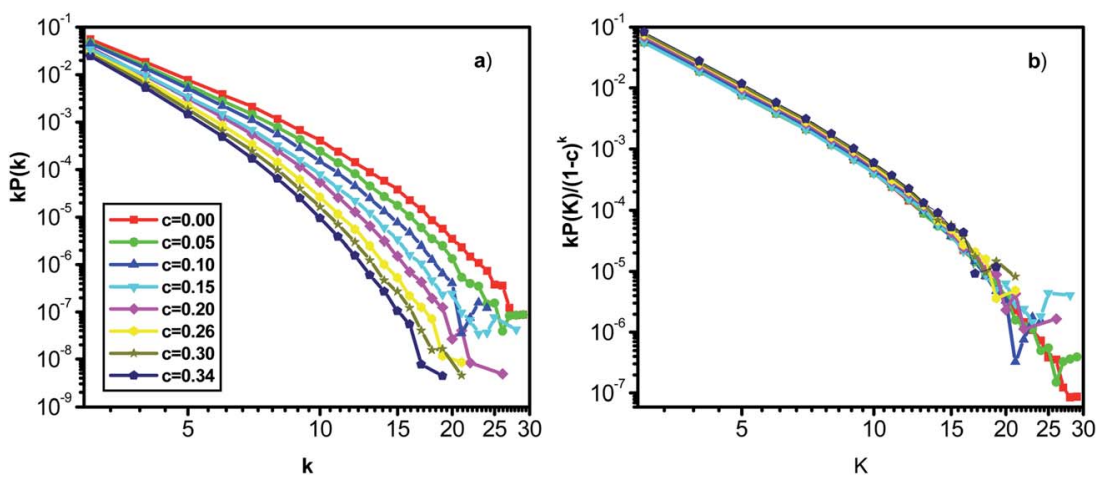

Fig. 2 (a) The probability that a bead takes place in a cooperative loop of the length $k$ versus the length of the loop $k$ for various concentrations of obstacles $c$. (b) The reduced probability $k \cdot P(k) /(1-c)^{k}$ versus the length of the loop $k$ for various concentrations of obstacles $c$ (see text for details).

twice going from the system without obstacles to the highest considered obstacle density $(c=0.34)$. These changes in the probability of the participation in a cooperative loop result in changes in the liquid mobility. In other words, the increase of the obstacle concentration makes the hydrodynamic interactions induced by the cooperative loop strongly screened by obstacles, which is expected and manifests in the decrease of the effective coordination number.

The total probability of a bead motion $P_{\text {tot }}$ could also be defined as the total number of cooperative movements $N_{\text {tot }}$ performed by all (mobile and immobile) beads in the entire system divided by the number of simulation steps $R$ and by the size of the system (in 2-D system represented by $L^{2}$ ):

$$
P_{\text {tot }}=\frac{N_{\text {tot }}}{R L^{2}}
$$

To calculate the total probability of move for mobile particles only $P_{\text {tot_mov }}$, eqn (3) should be divided by the factor $1 /(1-c)$ in order to deal with moveable elements only. An introduction of this factor leads to a significant decrease of the total probability of the bead movement $P_{\text {tot_mov }}$ with increasing obstacle

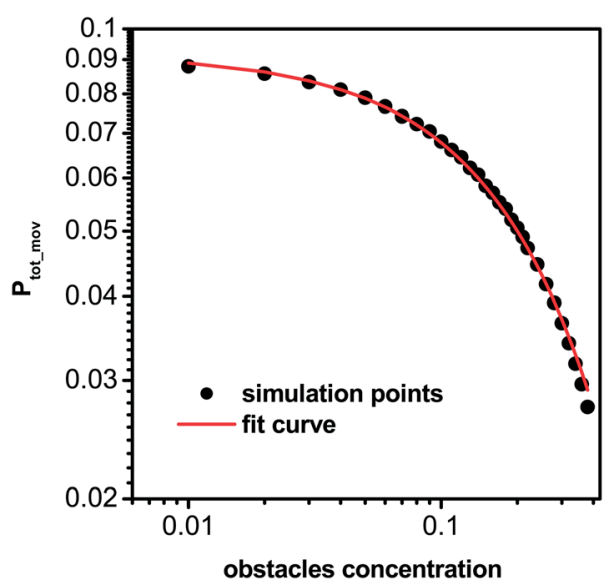

Fig. 3 The total probability of the bead movement in a cooperative loop $P_{\text {tot_mov }}$ for movable particles only (see text for details) as a function of the concentration of obstacles $c$. concentration, which is illustrated in Fig. 3. The behavior of the probability $P_{\text {tot_mov }}$ as a function of the obstacle concentration in the considered range of the obstacles' density $c$ can be easily approximated by the following function:

$$
P_{\text {tot_mov }}(c)=a \mathrm{e}^{-b c}
$$

where the fitting parameters are: $a=0.0916 \pm 0.003$ and $b=$ $3.02 \pm 0.02$. It implies that the mean waiting time $1 / P_{\text {tot_mov }}$ increases exponentially with the increasing concentration of obstacles.

\section{B. Dynamic properties}

It is particularly interesting to analyze the local dynamics, the dynamics that concern only a given lattice point in the investigated system, because quantities averaged over all beads in the system usually obscure the details of the local behavior. We started this analysis by defining the probability of a single bead cooperative move:

$$
P_{\text {bead }}=\frac{N_{\text {bead }}}{R}
$$

where $N_{\text {bead }}$ is the total number of accepted cooperative movements of a given lattice site. The reciprocal of this probability $1 / P_{\text {bead }}$ corresponds to the waiting time for the move of a given lattice site. The information about changes in the local dynamics of the system with the concentration of obstacles can be obtained from the distribution of the probability that a single bead performs a cooperative move in the regarded lattice site. Fig. 4 depicts the distribution of the probability of a molecule (a bead) cooperative move $P_{\text {bead }}$ for various concentrations of obstacles $c$. For the system without obstacles one peak can be found only for the probability near 0.09 . This behavior is rather obvious as the system is homogeneous and no lattice site and no direction are distinguished. The situation changes when immobile obstacles are introduced into the system. Their presence leads to a heterogeneous nature of the environment, which results in a broad distribution of the probability of a cooperative move: from the probability 0.01 to 0.09 . The distribution of the move probability is not continuous and 


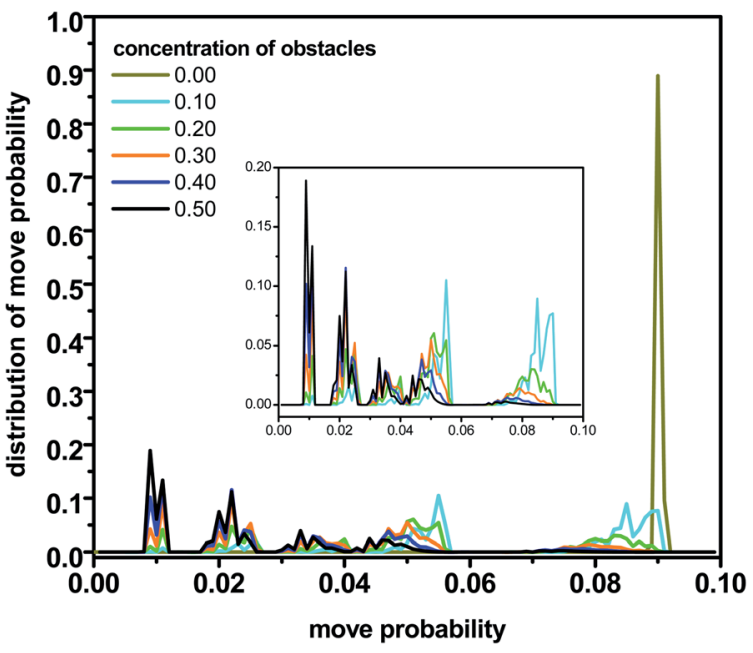

Fig. 4 The distribution of the probability of a bead cooperative move $P_{\text {bead }}$ for various concentrations of obstacles $c$. The inset shows the distribution of the probability of bead cooperative move for systems with non-zero concentration of obstacles.

consists of some widely distributed peaks. This kind of behavior is connected with the presence of obstacles but one has to keep in mind that our model is discrete. The distribution of the move probability shifts towards lower probability with the increasing concentration of obstacles, which was expected. The confirmation of this distribution one can find when studying a part of the simulated system as shown in Fig. 5 . The probability of the cooperative move in a given lattice site is marked by colors (in general dark colors correspond to lower mobility and light ones to the higher one). The hydrodynamic nature of the system in motion is revealed in this figure: the presence of the obstacles induces the regions of the slower motion near the obstacles. Thus, the distribution of the waiting time for a single bead at a given place (which shows how many time beads spend in a given lattice site before moving to another, neighboring site) is also broad. The map of the probability of cooperative motion shown in Fig. 5 is fully consistent with the distribution of the

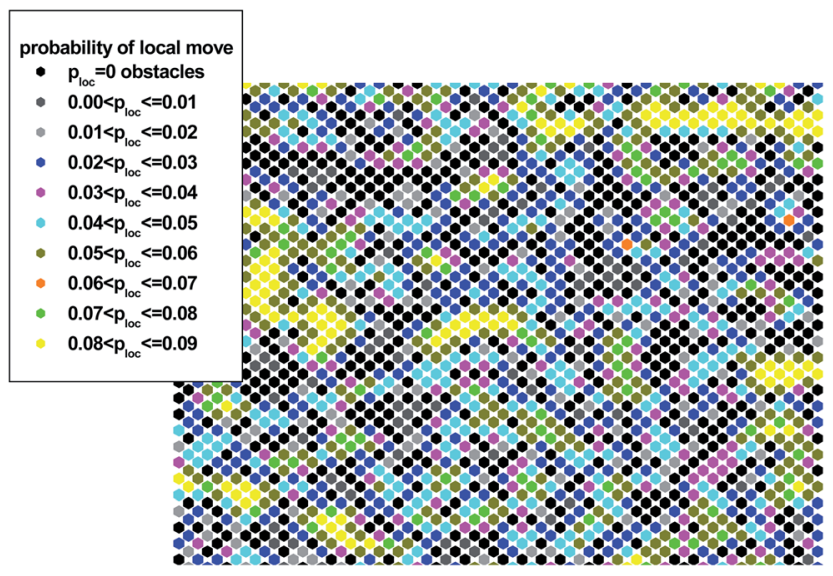

Fig. 5 The probability of the motion in a system with the concentration of obstacles $c \approx 0.30$. The assignments of colors to the probabilities are given in the inset. probability of motion presented in Fig. 4. It was found that a local environment strongly influences this probability. For a simple liquid a monomodal distribution of the move probability is observed, while for more complex systems, the presence of obstacles creates additional probability peaks. Moreover two trends are clearly visible: (i) probability peaks shift to lower values and simultaneously broaden with the increasing obstacle concentration and (ii) the distribution of the move probability is shifted towards low values (peaks at lower values become stronger) with the increasing obstacle concentration.

The time that mobile elements spend in a lattice site before leaving it is the next interesting quantity especially in the light of the CTRW model. The waiting time distribution is obtained by monitoring the waiting time of mobile elements along their trajectory. Fig. 6a depicts the waiting time distribution for several values of the obstacle concentration $c$. The distribution is quite broad even for the homogeneous case $(c=0)$, and becomes broader for heterogeneous medium, which has been already illustrated in Fig. 4 and 5. The waiting time is longer for the places that have several obstacles as neighbors and shorter for places where the obstacles are distant. Fig. $6 \mathrm{~b}$ shows the comparison of the average waiting time $1 / P_{\text {tot }}$ with the waiting time calculated from the waiting time distribution along trajectories. One can observe that values determined via both methods are in good agreement.

The next problem is related to the diffusion in the studied model systems. Positions of all mobile beads in the simulated systems were monitored during the entire simulation run by the mean-squared displacement (MSD) of the beads, defined as

$$
\left\langle\Delta r^{2}(t)\right\rangle=\frac{1}{n} \sum_{i=1}^{n}\left[\vec{r}_{i}(t)-\vec{r}_{i}(0)\right]^{2}
$$

where $\vec{r}_{i}(t)$ are the space coordinates of the $i$ th bead at time $t$ and $n$ is the number of moving elements. In the case of the normal (Fickian) diffusion the mean square displacement is proportional to the time $t$. The diffusion coefficient $D$ in twodimensional systems is described by the Einstein relationship
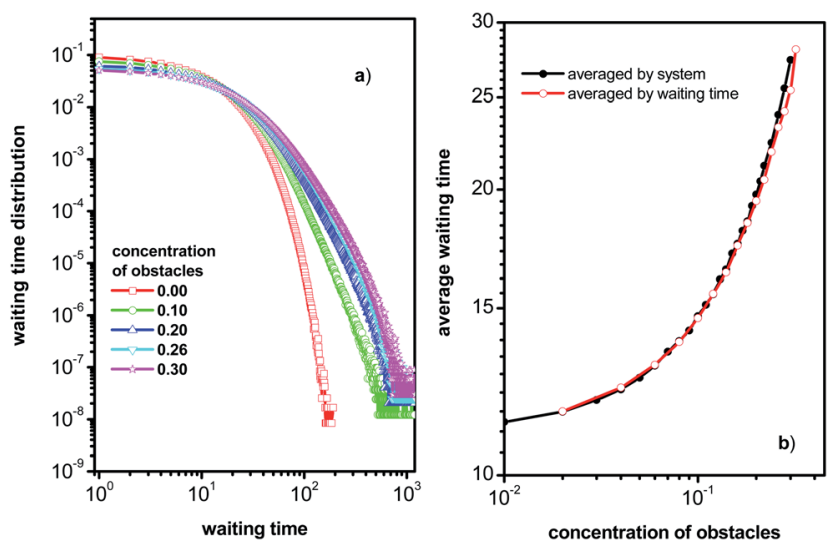

Fig. 6 (a) Waiting time distribution along the trajectory for several concentrations of obstacles. (b) The comparison of the mean waiting time obtained by using the distribution along the trajectory and the distribution of waiting time in given lattice sites (see text for details). 


$$
\left\langle\Delta r^{2}\right\rangle=4 D t
$$

In disordered systems this law is not valid and the anomalous diffusion appears. Near the percolation threshold one has the following formula:

$$
\left\langle\Delta r^{2}\right\rangle \approx t^{\alpha} \approx t^{2 / d_{\mathrm{w}}}
$$

where $\alpha=2 / d_{\mathrm{w}}$ is the anomalous diffusion exponent. The diffusion is hindered in these conditions and one can find $\alpha \leq 1$ or $d_{\mathrm{w}} \geq 2 .^{28,30}$ Other important transport characteristics of the investigated system can be obtained from the analysis of the velocity autocorrelation function (VACF). The negative values in the VACF indicate a persistent tendency of the diffusing molecules to invert their direction of motion and thus a tendency to stay localized. To investigate the existence of negative values in the VACF we estimated the contribution of the individual molecules through time averages

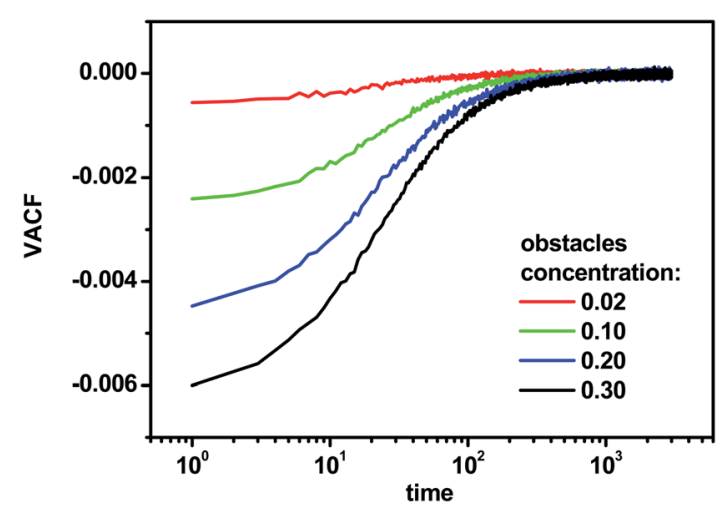

Fig. 7 Negative part of velocity autocorrelation function (VACF) for the two-dimensional DLL model. Mean square displacement $\left\langle\Delta r^{2}(t)\right\rangle$ as a function of time for various concentrations of obstacles.

$$
\operatorname{cor}_{i}(n)=\frac{1}{N-n} \sum_{k=0}^{N-n-1} \overrightarrow{u_{i}}(k) \cdot \overrightarrow{u_{i}}(k+n)
$$

where $n$ corresponds to the lag time and $N$ corresponds to the length of the time series. Finally, the VACF was calculated as the average over the individual contributions

$$
\operatorname{cor}(n)=\frac{1}{M} \sum_{j=1}^{M} \operatorname{cor}_{j}(n)
$$

where $M$ is the total number of investigated molecules. The results for various obstacle concentrations are presented in Fig. 7, where the VACF has been normalized in the way that $\operatorname{cor}(0)=1$. The VACF entered the region of anticorrelation already after one simulation step and this region has only been shown. The observed effect is similar to the results obtained for the Lorentz gas model ${ }^{69}$ where one can observe the increasing anticorrelation behavior with the increase of the concentration of obstacles.

Fig. 8a shows the mean square displacement as a function of time for some obstacle densities. One can observe the changes of $\left\langle\Delta r^{2}\right\rangle$ over a large time scale (above 8 decades): the upper curve (no obstacles) exhibits the exponent $\alpha=1$ in the entire time range while the other ones (the obstacle density above the percolation threshold) reach a plateau for longer times - the obstacles apparently divide the space into separated cages and the motion is limited. The systems with the obstacles below the percolation threshold exhibit three regimes but the conventional plot in Fig. 8a does not clearly show the changes of the diffusion behavior. Therefore, we have also plotted the simulation results as $\alpha=\frac{\mathrm{d}\left(\log \left(\Delta r^{2}(t)\right)\right)}{\mathrm{d}(\log (t))}$ versus $\log (t)$, which is presented in Fig. 8b. The diffusion at short times $\left(t \leq 5 \times 10^{1}\right)$ is always normal ( $\alpha$ is close to 1 ) in spite of the presence of obstacles (for the concentration of obstacles lower than the percolation threshold). One can observe that for short times $(t \leq$
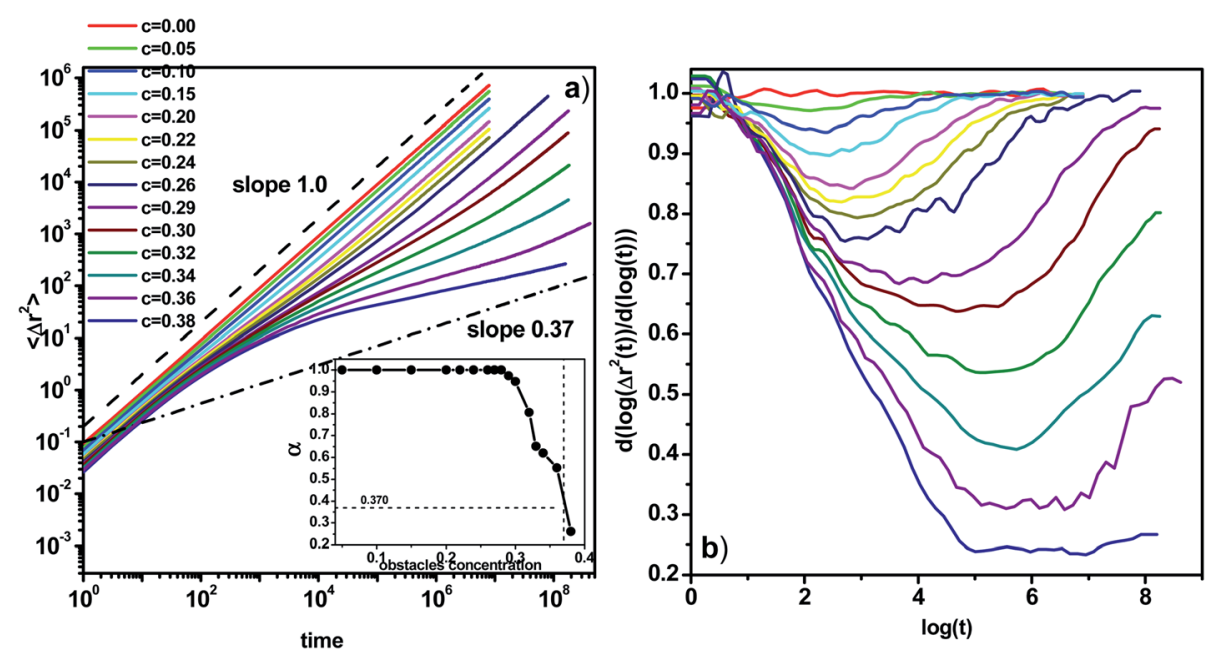

Fig. 8 (a) Mean square displacement $\left\langle\Delta r^{2}(t)\right\rangle$ as a function of time for various concentrations of obstacles. The inset indicates the exponent $\alpha$ for a long time as a function of the obstacle concentration c. (b) $\alpha=\frac{\mathrm{d}\left(\log \left(\Delta r^{2}(t)\right)\right)}{\mathrm{d}(\log (t))}$ as a function of logarithm of time for various obstacle concentrations (concentrations of obstacles are marked with the same colors as in (a)). 
$5 \times 10^{1}$ ) the mobility of moving particles systematically decreases with the increase of the obstacle concentration. This relates to the changes of a local arrangement of obstacles in the system when their concentration becomes higher and their influence on the short time (local) diffusion increases. For systems containing obstacles at intermediate times $\left(5 \times 10^{1}<\right.$ $t \leq 10^{4}$ to $10^{7}$ ) the diffusion is anomalous, while for long times it becomes normal again ( $\alpha$ is close to 1 ). This regime begins near $t=5 \times 10^{1}$, but its end shifts towards a longer time and the closer is the system to the critical density the faster is the shift. What is interesting is the fact that the curves which correspond to $c \geq 0.38$ (the curve for $c=0.38$ is only shown in Fig. $8 \mathrm{~b}$ for clarity) exhibit perfect plateaus over almost three decades. If we compare Fig. $8 \mathrm{a}$ and $\mathrm{b}$ we can observe a crossover to a normal diffusion at higher concentrations of obstacles $0.38<c<0.50$; for longer times the curve for $c=0.38$ scales with the exponent $a$ close to 0.25 .

Taking into consideration the above picture of the diffusion process one can introduce short time $D_{\mathrm{SH}}$ and long time $D_{\mathrm{L}}$ diffusion constants of mobile beads. Fig. 9 depicts the behavior of these quantities as a function of the obstacle concentration. One can find that the short time mobility described by the $D_{\mathrm{SH}}$

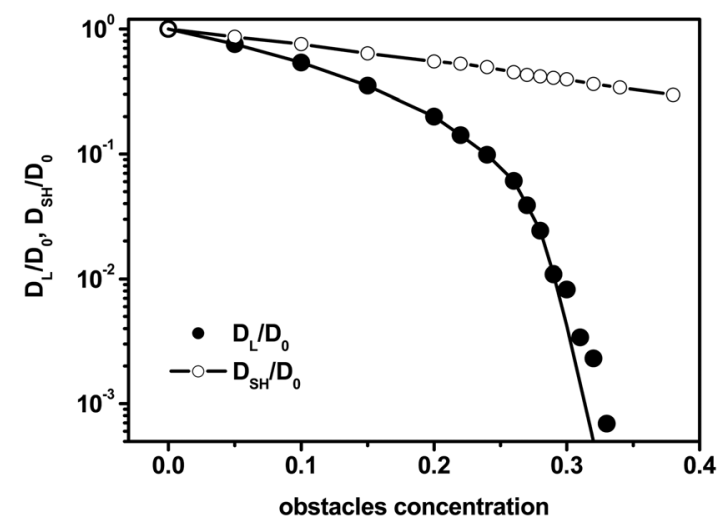

Fig. 9 Short time diffusion $D_{\mathrm{SH}}$ and long time diffusion $D_{\mathrm{LT}}$ coefficients as a function of the obstacle concentration $c$.

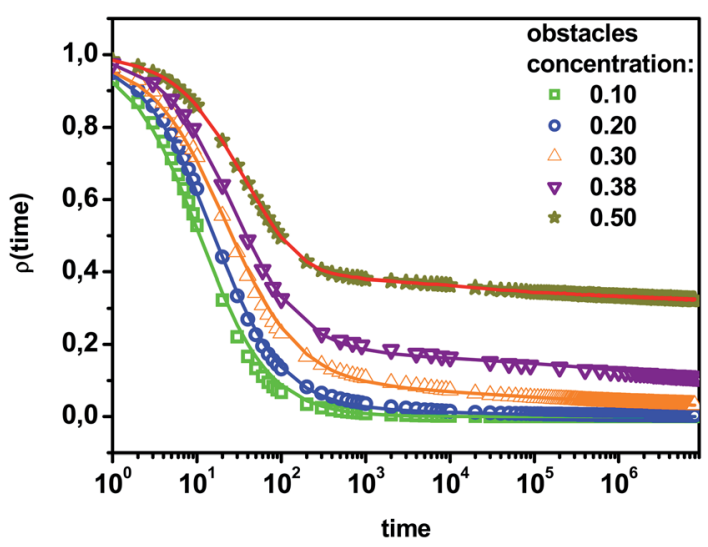

Fig. 10 The position correlation function $\rho(t)$ (see eqn (9) and text for details) for various concentrations of obstacles $c$. Continuous lines represent fitting curves obtained using eqn (10). constant decreases with the obstacle concentration exponentially $\left(D_{\mathrm{SH}}=D_{0} \exp (-a c)\right.$, where $D_{0}$ corresponds to the diffusion coefficient when the concentration of obstacles is equal to 0 ). The decrease of the long time diffusion coefficient $D_{\mathrm{L}}$ is much faster and has more complex character. We can confirm the differences between the short and long time dynamic behaviors of the system by studying the position correlation function of the mobile elements. It can give important information about the global dynamics in dense systems, where the movement of particles is highly correlated. This function is defined in the following way:

$$
\rho(t)=\frac{1}{n} \sum_{i=1}^{n} m_{i}(0) m_{i}(t)
$$

where $m_{i}(0)=1$ and $m_{i}(t)=1$ or 0 , depending whether or not the $i$ th bead occupied its original position (at $t=0$ ) and at a given time $t$ respectively. Fig. 10 shows the position correlation function calculated according to eqn (9) for various concentrations of obstacles. Observing this function one can distinguish two relaxation processes in the system. The fast relaxation process corresponds to the short time diffusion. The second process corresponds to a long time delay process connected with movement of the beads, which are hindered by trapping in cages. This process becomes visible for the concentration of obstacles $c \approx 0.20$. The existence of this plateau on the correlation function shows that in the considered range of concentrations more and more beads cannot be included into cooperative loops or are caged in closed enclaves if the concentration of obstacles increases. This effect is especially well visible at the concentration of obstacles 0.5 which corresponds to the ordinary percolation threshold on the triangular lattice,$^{70}$ where many beads are caged in a dead end branch or in closed enclaves. Thus, the behavior of the position correlation function that can be split into two processes based on quite different time scales confirms the influence of the local environment on the mean square displacement and the selfdiffusion with the increase of crowding.

\section{Dynamic behavior near the critical point: the percolation threshold}

Direct observation of the percolation transition is not easy using MSD because it is only the simplest quantity exhibiting anomalous long-time behavior. Deviations from the Fickian diffusion can be indicated by a parameter which is more sensitive than MSD: it is called the (first) non-Gaussian parameter (NGP) quantifying deviations from the Gaussian distribution ${ }^{71}$ which in the two-dimensional case looks as follows:

$$
\alpha_{2}(t)=\frac{\left\langle\Delta r^{4}(t)\right\rangle}{2\left\langle\Delta r^{2}(t)\right\rangle}-1
$$

The long time limit of $\alpha_{2}(t)$ is presented in Fig. 11. One can observe that the highest deviation from the Gaussian behavior at the long time is found for $c=0.38$. It suggests that one should expect the percolation point near $c=0.38 .^{72}$ Taking into consideration the above observations and based on the data 


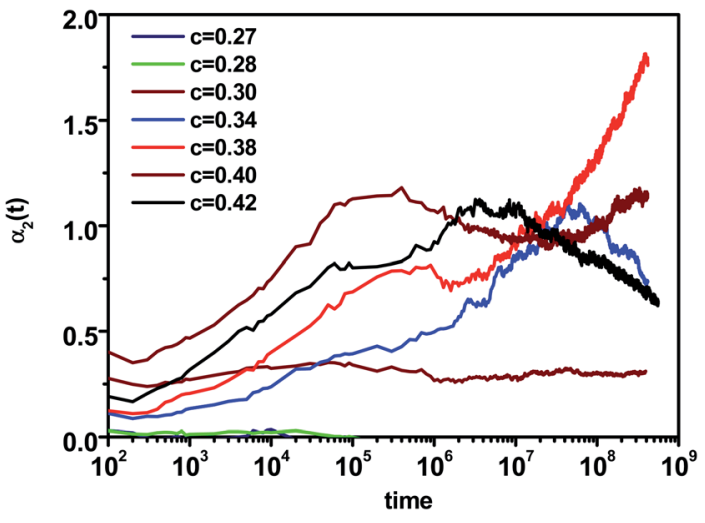

Fig. 11 The non-Gaussian parameter $\alpha_{2}(t)$ for various concentrations of obstacles $c$.

from Fig. 9 one can perform the scaling analysis of the diffusion coefficient $D$ (in the limit of the long times which were obtained by $\left.D=\left\langle\Delta r^{2}\right\rangle / t, t \rightarrow \infty\right)$ near the critical point $c=0.38$ as a function of the obstacle concentration, which is presented in Fig. 12. The diffusion coefficient follows the predicted scaling relationship $D \approx \varepsilon^{\mu}$ where $\varepsilon=\left|c-c_{\mathrm{c}}\right| / c_{\mathrm{c}}$ and $\mu \approx 4.34 .^{28,30,73}$ The critical density was found to be $c_{\mathrm{c}} \approx 0.371$ but one has to remember that the value of the diffusion coefficient is approximate.

The diffusion in a percolation cluster can be described in two different ways. In the first, one can restrict walks to the incipient infinite cluster at $p=p_{\mathrm{c}}$. In the second, one can study the diffusion in all clusters of the system unrestrictedly and this treatment corresponds to the applied herein model. Moreover, our case is similar to the high-density percolation problem ${ }^{74}$ which shares the same universality class as the ordinary lattice percolation. It means that for two dimensions the universal exponent describing the probability that a lattice site belongs to the percolation cluster is equal to $\beta=5 / 36$ and the universal exponent, which describes the divergence of the correlation length, near the percolation threshold is equal to $\nu=4 / 3$. Using the scaling analysis one can find that in our case the exponent

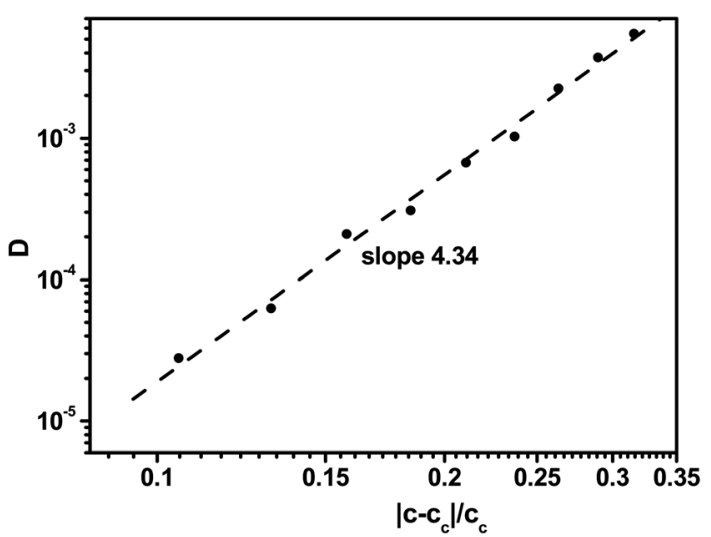

Fig. 12 Scaling behavior of the long-time diffusion coefficient $D$ as a function of separation parameter $\varepsilon=\left|c-c_{c}\right| / c_{c}$. $d_{\mathrm{w}}$ from eqn (8) (near the critical point) can be described by the formula: ${ }^{28,30}$

$$
d_{\mathrm{w}}=\frac{2+\mu / v-\beta / v}{1-\beta / 2 v}
$$

In our case $d_{\mathrm{w}} \approx 5.43$ and, therefore, it corresponds to the critical exponent $\alpha \approx 0.37$. Similar values of this exponent can be found in computer simulations of Lorentz gas, where $\alpha=$ 0.32 or 0.42 (ref. 72, 76 and 77) and in real experiments where $\alpha$ $=0.32$ or $0.39 .^{26,27}$ However, it has to be stressed that higher values of the subdiffusion exponent were also found in both simulations and experiments. ${ }^{37}$

Taking into consideration the results presented above a rough estimation of the percolation threshold can be done using the dependence of the exponent $\alpha$ on the obstacle concentration. In our model the percolation threshold corresponds to the concentration of obstacles $c_{\mathrm{c}} \approx 0.37-0.38$ (see the inset in Fig. 8a) and the concentration of liquid molecules at this threshold $p_{\mathrm{c}}$ is calculated as $p_{\mathrm{c}}=1-c_{\mathrm{c}} \approx 0.62-0.63$. One can observe quite good agreement between the percolation threshold obtained from the scaling law $D \approx \varepsilon^{\mu}$ and from direct measurements of the critical exponents $\alpha$. We have also used the so-called data collapsed technique, which is more sensitive than a single direct measurement of critical exponents to determine the exponent $\mu$ and the percolation threshold $c_{\mathrm{c}}$. This procedure is based on the fact that the mean squared displacement follows the scaling relationship ${ }^{26}$

$$
\left\langle\Delta r^{2}(t)\right\rangle=t^{2 / d_{\mathrm{w}}} f\left[\left(p-p_{\mathrm{c}}\right) t^{\left(d_{\mathrm{w}}-2\right) / \mu d_{\mathrm{w}}}\right] .
$$

The $f(x)$ is a scaling function given by

$$
f(x)= \begin{cases}x^{\mu} & \text { for } x \rightarrow \infty \\ \text { const } & \text { for } x \rightarrow 0 \\ (-x)^{-2 v+\beta} & \text { for } x \rightarrow-\infty\end{cases}
$$

Fig. 13a presents the expression $\left\langle\Delta r^{2}(t)\right\rangle t^{-2 / d_{\mathrm{w}}}$ as a function of $t\left(p-p_{\mathrm{c}}\right)^{\frac{\mu d_{\mathrm{w}}}{d_{\mathrm{w}}-2}}$ for various concentrations of liquid molecules $p$ (which implies various concentrations of obstacles as $c=1-p$ ). One can expect that the curves should approach a common slope $\left(d_{\mathrm{w}}-2\right) / d_{\mathrm{w}}$ for longer times. In this plot we used the exponent $\mu=4.34$, i.e. the one obtained from the scaling analysis of the diffusion coefficient and the percolation threshold $p_{\mathrm{c}} \approx 0.629\left(c_{\mathrm{c}} \approx 0.371\right)$. One can observe that the data collapse onto a single curve for longer times with deviations for short times, which is expected, because in this time region the system is far from the asymptotic behavior. The common straight line corresponds to a power law function with the slope $\left(d_{\mathrm{w}}-2\right) / d_{\mathrm{w}}=0.632$. Fig. 13b shows $\left\langle\Delta r^{2}(t)\right\rangle t^{-2 / d_{\mathrm{w}}}$ as a function of $t\left(p-p_{\mathrm{c}}\right)^{\frac{\mu d_{\mathrm{w}}}{d_{\mathrm{w}}-2}}$ for $x \rightarrow-\infty$ in eqn (15). In this case we have also obtained an expected behavior too, i.e. a common straight line. But the slope of this common line is -0.238 , which differs significantly from that expected using $\left(d_{\mathrm{w}}-2\right)(-2 \nu+\beta) / d_{\mathrm{w}} \mu \approx$ -0.352 . We cannot explain the reasons for this discrepancy but such behavior of scaling confirms that the percolation threshold was determined correctly. The above analysis 

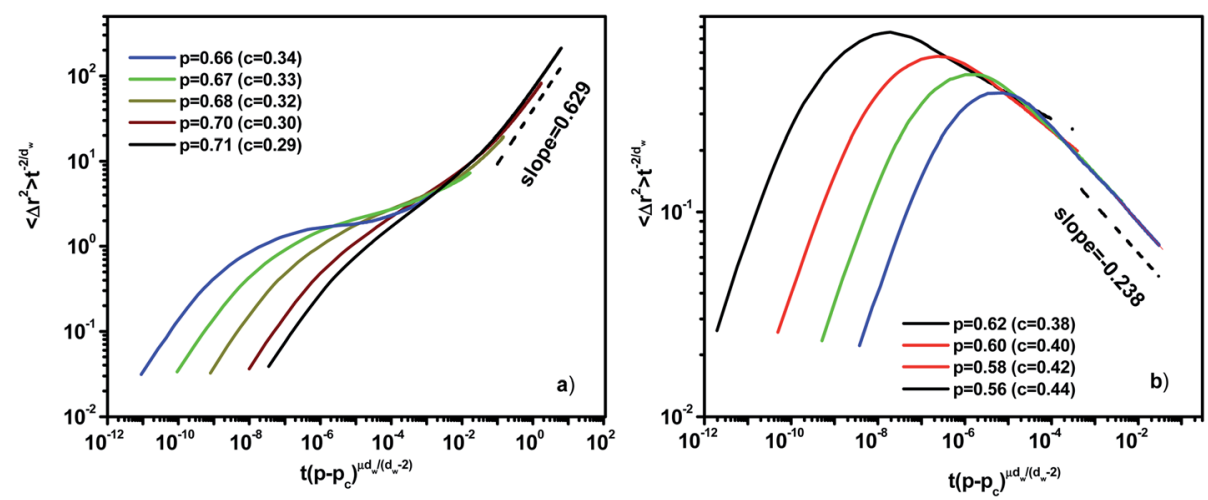

Fig. 13 The scaling function $\left\langle\Delta r^{2}(t)\right\rangle t^{-2 / d_{w}}$ as a function of $t\left(p-p_{c}\right)^{\frac{\mu d_{w}}{d_{w}-2}}$ for various concentrations of liquid $p$ (a) for $x \rightarrow \infty$ and (b) $x \rightarrow-\infty$ see eqn (15).

indicates that both the exponent $\mu=4.34$ calculated and the percolation threshold $c_{\mathrm{c}} \approx 0.371$ determined from the scaling analysis of the diffusion coefficient are rather appropriate values. Therefore, it is possible that the scaling behavior in our model is different from that of a single particle motion on the percolation clusters.

The next problem concerns the universality of the percolation phenomenon in our model system. The coefficient $D$ scales well as $\varepsilon^{\mu}$ with $\mu=4.34$ as shown in Fig. 12 . The exponent obtained in our simulations $\mu=4.34$ deviates considerably from the theoretical value 1.31 found for the $2 \mathrm{D}$ lattice system in which all the clusters of the system are taken into consideration unrestrictedly. The problem with the universality of the exponent $\mu$ and its dependency on the transport mechanism and on the matrix type was already reported over 25 years ago. ${ }^{75}$ The problem with no-universality of the $\mu$ exponent in 2D simulations was reported later in ref. 50, when the Discontinuous Molecular Dynamic (DMD) simulations and the spatial tessellation were used to investigate the effect of polydispersity in obstacle size on the solute diffusion. On the other hand perfect agreement between the expected scaling behavior of $\mu$ and simulation results in the case of the two-dimensional Lorentz model was reported. ${ }^{33}$ We cannot conclusively determine whether the exponent $\mu$ is universal. The transport mechanism in our model is completely different from the continuous model represented by the Lorentz model, the DMD model and from models based on a lattice. ${ }^{44}$ The movement of beads in our simulations is highly correlated and this way the mobility of the beads is strongly connected (hydrodynamics) with positions and the concentration of obstacles. This situation leads to specific changes of diffusivity in the system and consequently to higher values of the exponent $\mu$. This is proved by data presented in Fig. 5 which shows a typical part of the simulation system where the concentration of obstacles $c>0.3$. One can observe that the considered area contains enclaves where some beads move fast but the mobility of the most elements is strongly limited. The system percolates in the sense of the percolation cluster analysis but the motion in the system is determined by the mobility of the slowest elements. In other words, the system besides the real immobile obstacles contains also considerably less mobile molecules of liquids, which only very rarely can take place in the motion and therefore they play a crucial role in the movement of liquid. One should consider them as some kinds of fluctuating obstacles; in our opinion this phenomenon corresponds to the presence of small holes in the continuous-space system considered by Halperin $e t$ al. ${ }^{44}$

The next problem concerns the question how does the percolation threshold obtained by us can be related to other results obtained within the frame of a continuous model. It is especially interesting, as it is well known that a triangular lattice mimics a continuous two-dimensional space - the filling of the space by discs is the same for this lattice and for the continuous space. In order to make such a comparison possible one has to redefine the concentration on a triangular lattice. This new definition better corresponds to a continuous space and is calculated as the ratio of the total surface of disks representing beads to the total area of the considered system. The concentration based on this definition will be denoted by $\Phi$. In this convention the maximum possible concentration corresponds to the close packing of disks and it is approximately equal to 0.9069 . Taking into account this value one can find out that our percolation threshold $c_{\mathrm{c}} \approx 0.37-0.38$ corresponds to $\Phi_{\mathrm{c}} \approx$ $0.335-0.345$. This value is located between $\Phi_{\mathrm{c}}=0.22$ found by Sung and Yethiraj ${ }^{40}$ and the value $\Phi_{\mathrm{c}}=0.356$ obtained by Bauer and coworkers using the Lorentz gas model. ${ }^{33}$

\section{Conclusions}

In this work we have simulated the dynamic properties of a liquid in the presence of obstacles. A high density characterizes a liquid environment and consequently the movement of particles has to be highly correlated. Investigation of this type of system is particularly important from the cognitive point of view and for practical reasons, because such systems are very popular in nature. In order to accomplish this task, we used the DLL model, which easily allows incorporation of interactions between the solute (liquid) and the obstacles. The motion in this model is realized in a way of cooperative loops, which more resembles a real liquid in comparison with other models, where the motion is determined randomly from an arbitrary assumed distribution. The excluded volume was the only potential included in our model. Moreover, the influence of 
hydrodynamic interactions can also be taken into consideration in our model.

We analyzed the behavior of the basic parameters of the DLL model in the presence of obstacles. The concentration of obstacles affects the dynamics of the system (which was expected) by the modification of the distribution of cooperative movement loops. It was found that the main factor, which is responsible for the cooperative move loop distribution for a given concentration of obstacles, is the so-called effective coordination number. This quantity decides on the conductivity between near neighbor lattice sites and it decreases with the obstacle concentration $c$ like $1-c$ in the investigated range of obstacle concentrations. Our treatment based on the DLL model allows the exact investigation of the local behavior of the system, and we determined the distribution probability of the bead move in a given lattice site (depending on this site's neighborhood).

The analysis of the waiting time distribution along the trajectory indicates that this distribution does not display a power-law behavior and thus one can conclude that the CTRW model is not consistent with the DLL model (when the obstacles are present). The study of the dynamics generated by the DLL model, i.e. the diffusion and the position correlation function, indicated the existence of two relaxation processes associated with the movement in the presence of obstacles. A short relaxation time is associated with the fast beads, located far from obstacles, while the slow process corresponds to motion of beads with the reduced mobility as a result of the location near obstacles or remaining in cages arranged by obstacles. Moreover, the simulations show a transition from a normal to an anomalous diffusion of beads as the concentration of obstacles increases.

We show that in our 2D model the percolation threshold occurs at the obstacle concentration $c=0.37-0.38$. Simple comparison of our results with the results obtained using the continuous model in $2 \mathrm{D}$ places them between the results obtained for the Lorentz gas model ${ }^{33}$ and the results found using the discontinuous molecular dynamic model. ${ }^{40}$ It was also found that the diffusion coefficient for a long time near the percolation threshold scales as $D \approx \varepsilon^{\mu}$ where $\varepsilon=\left|c-c_{\mathrm{c}}\right| / c_{\mathrm{c}}$ with $\mu \approx 4.34$. This value of the exponent $\mu$ is different from the pervious prediction obtained from the percolation theory and simulations, ${ }^{50}$ where $\mu=1.31$. This probably results from the completely different transport mechanism in our model and mechanisms proposed in continuous models (represented by the Lorentz model or by the DMD model) and in models based on a lattice. ${ }^{33}$ The presence of a very high value of the exponent $\mu$ can explain why the exponent $\alpha$ determined in experiments is considerably lower than 0.6-0.7. Thus, the scenario based on the DLL model can be closer to reality than other models, especially in membranes or cells.

Our simulation results indicate that the DLL model can be effectively used in a dense system, where all lattice sites are occupied by moving objects or obstacles, i.e. when the environment is crowded. The DLL model also allows the introduction of the dynamics into the system in a natural way based on cooperative concepts with a good time scale connected with one MC step. Simulations based on the DLL model enable easy observations of the local and global dynamics. Moreover, our treatment can be directly applied to other systems with a crowded environment, which contains obstacles or traps of various shapes and different levels of dispersion.

\section{Acknowledgements}

The authors gratefully acknowledge the computational grant 77/2012 from the Supercomputing and Networking Center (PCSS) in Poznan, Poland. The computations were also carried out using the computer cluster at the Computing Center of the Department of Chemistry at the University of Warsaw, Poland.

\section{References}

1 R. J. Cherry, P. R. Smith, I. E. Morison and N. Fernandez, FEBS Lett., 1988, 430, 88.

2 M. Küntz and P. Lavallée, J. Phys. D: Appl. Phys., 2001, 34, 2547.

3 F. Varnik, J. Baschangel and K. Binder, J. Non-Cryst. Solids, 2002, 307-310, 524.

4 M. Takuyama, Y. Enomoto and I. Oppenheim, Phys. Rev. E: Stat. Phys., Plasmas, Fluids, Relat. Interdiscip. Top., 1997, 56, 2302.

5 D. C. Schlosser, K. Morgenstern, L. H. Verheij, G. Rosenfeld, F. Besenbacher and G. Cosma, Surf. Sci., 2000, 465, 19.

6 S. Condamin, V. Tejedor, R. Voituriez, O. Bénichou and J. Klafter, Proc. Natl. Acad. Sci. U. S. A., 2008, 105, 5675.

7 D. S. Banks and C. Fradin, Biophys. J., 2005, 89, 2960.

8 M. J. Saxton, Biophys. J., 2007, 92, 1178.

9 G. Guglas, C. Kalla and M. Weiss, FEBS Lett., 2008, 581, 5094.

10 A. M. S. Niehaus, D. G. Vlachos, J. S. Edwards, P. Plechac and R. Tribe, Biophys. J., 2008, 94, 1551.

11 J. A. Dix and A. S. Verkman, Annu. Rev. Biophys., 2008, 37, 247.

12 H.-X. Zhou, G. Rivas and A. P. Minton, Annu. Rev. Biophys, 2008, 37, 375.

13 D. Fanelli and A. J. McKane, Phys. Rev. E: Stat. Phys., Plasmas, Fluids, Relat. Interdiscip. Top., 2010, 82, 021113.

14 D. Holcman, N. Hoze and Z. Schuss, Phys. Rev. E: Stat. Phys., Plasmas, Fluids, Relat. Interdiscip. Top., 2011, 84, 021906.

15 R. J. Ellis, Trends Biochem. Sci., 2001, 26, 597.

16 M. Długosz and J. Trylska, BMC Biophys., 2011, 4, 3.

17 S. Ramadurai, A. Holt, V. Krasnikov, G. van den Bogaart, J. A. Killian and B. Poolman, J. Am. Chem. Soc., 2009, 131, 12650.

18 J. Szymanski and M. Weiss, Phys. Rev. Lett., 2009, 103, 038102.

19 M. Wachsmuth, W. Waldeck and J. Langowski, J. Mol. Biol., 2000, 298, 677.

20 M. R. Horton, F. Höfling, J. O. Rädler and T. Franosch, Soft Matter, 2010, 6, 2648.

21 G. Lindblom and G. Orädd, Biochim. Biophys. Acta, 2009, 1788, 234.

22 C. Selle, F. Rückerl, D. S. Martin, M. B. Forstner and J. A. Käs, Phys. Chem. Chem. Phys., 2004, 6, 5535.

23 M. Vrljic, S. Y. Nishimura, S. Brasselet, W. E. Moerner and H. M. McConnell, Biophys. J., 2002, 83, 2681. 
24 M. Renner, Y. Domanov, F. Sandrin, I. Izeddin, P. Bassereau and A. Triller, PLoS One, 2011, 6, e25731.

25 F. L. Brown, D. M. Leitner, J. A. McCammon and K. R. Wilson, Biophys. J., 2000, 78, 2257.

26 I. Bronstein, Y. Israel, E. Kepten, S. Mai, Y. Tal-Shav, E. Barkai and Y. Garini, Phys. Rev. Lett., 2009, 103, 018102.

27 S. C. Weber, A. J. Spakowitz and J. A. Theriot, Phys. Rev. Lett., 2010, 104, 238102.

28 D. Ben-Avraham and S. Havlin, Diffusion and reactions in fractals and disordered systems, Cambridge University Press, Cambridge, 2000.

29 P. F. F. Almeida and W. L. C. Vaz, in Handbook of Biological Physics, Structure and Dynamics of Membranes: From Cells to Vesicles, ed. R. Lipovsky and E. Sackman, Elsevier North Holland, Amsterdam, 1995, vol. 1.

30 J. P. Bouchaud and A. Georges, Phys. Rep., 1990, 195, 127.

31 A. P. Minton, J. Cell Sci., 2006, 118, 2863.

32 A. Kammerer, F. Höfling and T. Franosch, Europhys. Lett., 2008, 84, 66002.

33 T. Bauer, F. Höfling, T. Munk, E. Frey and T. Franosch, Eur. Phys. J.: Spec. Top., 2010, 189, 103.

34 F. Hofling, K.-U. Bamberg and T. Franosch, Soft Matter, 2011, 7, 1358.

35 I. M. Sokolov, Soft Matter, 2012, 8, 9043.

36 M. J. Saxton, Biophys. J., 2012, 103, 2411.

37 F. Höfling and T. Franosch, Rep. Prog. Phys., 2013, 76, 046602.

38 M. J. Saxton, Biophys. J., 1993, 64, 1053.

39 M. J. Saxton, Biophys. J., 1996, 70, 1250.

40 J. S. Sung and A. Yethiray, Phys. Rev. Lett., 2006, 96, 228103.

41 M. J. Saxton, Biophys. J., 2001, 81, 2226.

$42 \mathrm{H}$. Berry and H. Chaté, arXiv:1103.2206v1, 2011.

43 R. Metzler and J. Klafter, Phys. Rep., 2010, 339, 1.

44 B. I. Halperin, S. Feng and P. N. Sen, Phys. Rev. Lett., 1985, 54, 2391.

45 B. J. Sung and A. Yethiraj, J. Phys. Chem. B, 2008, 112, 143.

46 B. J. Sung and A. Yethiraj, J. Chem. Phys., 2008, 128, 054702.

47 T. Voigtmann and J. Horbach, Phys. Rev. Lett., 2009, 103, 205901.

48 K. Kim, K. Miyazaki and S. Saito, Europhys. Lett., 2009, 88, 36002.

49 J. Kurzidim, D. Coslovich and G. Kahl, Phys. Rev. Lett., 2009, 103, 138303.

50 H. W. Cho, G. Kwon, B. J. Sung and A. Yethiraj, Phys. Rev. Lett., 2012, 109, 155901.

51 T. O. E. Skinner, S. K. Schnyder, D. G. A. L. Aarts, J. Horbach and R. P. A. Dullens, Phys. Rev. Lett., 2013, 111, 128301.
52 G. R. Kneller, K. Baczynski and M. Pasenkiewicz-Gierula, J. Chem. Phys., 2011, 135, 3651800.

53 M. Javanainen, H. Hammaren, L. Monticelli, J.-H. Jeon, M. S. Miettinen, H. Martinez-Seara, R. Metzler and I. Vattulainen, Faraday Discuss., 2013, 161, 397.

54 J. E. Goose and M. S. P. Sansom, PLOS Comput. Biol., 2013, 9, e1003033.

55 P. Polanowski and T. Pakula, J. Chem. Phys., 2003, 118, 11139.

56 P. Polanowski and T. Pakula, J. Chem. Phys., 2004, 120, 6306.

57 P. G. de Gennes, Scaling Concepts in Polymer Physics, Cornell University Press, Ithaca, NY, 1979.

58 P. G. Saffman and M. Delbrück, Proc. Natl. Acad. Sci. U. S. A., 1975, 72, 3111.

59 S. J. Bussell, D. L. Koch and D. A. Hammer, Biophys. J., 1995, 68, 1836.

60 F. L. H. Brown, Q. Rev. Biophys., 2011, 44, 391.

61 P. Polanowski and Z. Koza, Phys. Rev. E: Stat. Phys., Plasmas, Fluids, Relat. Interdiscip. Top., 2006, 74, 36103.

62 P. Polanowski and T. Pakula, J. Chem. Phys., 2002, 117, 4022.

$63 \mathrm{H}$. Gao, P. Polanowski and K. Matyjaszewski, Macromolecules, 2009, 42, 5929.

64 P. Polanowski, J. K. Jeszka and K. Matyjaszewski, Polymer, 2010, 51, 6084.

65 P. Polanowski, J. K. Jeszka, W. Li and K. Matyjaszewski, Polymer, 2011, 52, 5092.

66 P. Polanowski, J. K. Jeszka and K. Matyjaszewski, Polymer, 2013, 54, 1979.

67 B. D. Hughes, Random Walk and Random Environments, Clarendon, Oxford, 1995.

68 T. Pakula, Simulation on the Completely Occupied Lattices, in Simulation Methods for Polymers, ed. M. Kotelyanskii and D. N. Theodorou, Marcel Dekker, New York, Basel, 2004.

69 F. Höfling and T. Franosch, Phys. Rev. Lett., 2207, 98, 140601.

70 D. Stauffer and A. Aharony, Introduction to Percolation Theory, Taylor and Francis, London, 1994.

71 J. P. Boon and D. Yip, Molecular Hydrodynamics, Dover, New York, 1991.

72 F. Höfling, T. Franosch and E. Frey, Phys. Rev. Lett., 2006, 96, 165901.

73 M. P. M. den Nijs, J. Phys. A: Math. Gen., 1979, 12, 1857.

74 G. R. Reich and P. L. Leath, J. Stat. Phys., 1978, 19, 611.

75 I. A. Park and J. M. D. MacElroy, Mol. Simul., 1989, 2, 105.

76 F. Höfling, T. Munk, E. Frey and T. Franosch, J. Chem. Phys., 2008, 128, 164517.

77 M. Spanner, F. Höfling, G. E. Schröder-Turk, K. Meck and T. Franosch, J. Phys.: Condens. Matter, 2011, 23, 234120. 